\title{
DIURNAL VARIATION IN WINGATE TEST PERFORMANCES: INFLUENCE OF ACTIVE WARM-UP
}

\author{
Nizar Souissi, ${ }^{1}$ Tarak Driss, ${ }^{2}$ Karim Chamari, ${ }^{1}$ Henry Vandewalle, ${ }^{3}$ Damien \\ Davenne, ${ }^{4}$ Abdelkader Gam, ${ }^{1}$ Jean-Robert Fillard, ${ }^{5}$ and Eric Jousselin ${ }^{5}$ \\ ${ }^{1}$ Research Laboratory "Sports Performance Optimizaion," National Centre of Medicine \\ and Science in Sport (CNMSS), Tunis, Tunisia \\ ${ }^{2}$ Centre de Recherche sur le Sport et le Mouvement, UFR STAPS, Université Paris Ouest \\ Nanterre - La Défense, France \\ ${ }^{3}$ Service d'Explorations Fonctionnelles et de Médecine du Sport, Bobigny, France \\ ${ }_{4}^{4}$ INSERM, Caen, France; Université de Caen, Caen, France \\ ${ }^{5}$ INSEP, Département Médical, Paris, France
}

The purpose of the present study was to examine the effects of active warm-up duration on the diurnal fluctuations in anaerobic performances. Twelve physical education students performed a medical stress test (progressive test up to exhaustion) and four Wingate tests (measurement of peak power $\left[\mathrm{P}_{\text {peak }}\right]$, mean power $\left[\mathrm{P}_{\text {mean }}\right]$, and fatigue index during an all-out $30 \mathrm{~s}$ cycling exercise). The tests were performed in separate sessions (minimum interval $=36 \mathrm{~h}$ ) in a balanced and randomized design at 08:00 and 18:00 h, either after a $5 \mathrm{~min}(5-\mathrm{AWU})$ or a $15 \mathrm{~min}$ active warm-up (15AWU). AWU consisted of pedaling at $50 \%$ of the power output at the last stage of the stress exhausting test. Rectal temperature was collected throughout the sessions. A two-way ANOVA (warm-up $\times$ time of day) revealed a significant interaction for $\mathrm{P}_{\text {peak }}$ $\left(\mathrm{F}_{(1.11)}=6.48, p<0.05\right)$ and $\mathrm{P}_{\text {mean }}\left(\mathrm{F}_{(1.11)}=5.84, p<0.05\right)$ : the time-of-day effect was significant $(p<0.001)$ in contrast with the effect of warm-up duration $(p>$ 0.05). $P_{\text {peak }}$ and $P_{\text {mean }}$ improved significantly from morning to afternoon after both 5-AWU and 15-AWU, but the effect of warm-up duration was significant in the morning only. Indeed, the values of $\mathrm{P}_{\text {peak }}$ or $\mathrm{P}_{\text {mean }}$ were the same after both warmup protocols in the afternoon. For rectal temperature, there was no interaction between time-of-day and warm-up duration. Rectal temperature before and after both the warm-up protocols was higher in the afternoon, and the effect of warm-up duration on temperature was similar at 08:00 and 18:00 h. In conclusion, the interpretation of the results of the anaerobic performance tests should take into account time-of-day and warm-up procedures. Longer warm-up protocols are

Address correspondence to Dr. Nizar Souissi, Institut Supérieur de Sport et de l'Education Physique de Ksar Saïd, Manouba, Tunis, 2010, Tunisie; Tel.: 0021696818 633; E-mail: n_souissi@yahoo.fr 
recommended in the morning to minimize the diurnal fluctuations of anaerobic performances. (Author correspondence: n_souissi@yahoo.fr)

Keywords Circadian rhythm; Temperature; Anaerobic performance; Cycling sprint; Wingate test; Muscle power

\section{INTRODUCTION}

The effects of the circadian rhythm and time of day on short-term maximal exercises ( $\leq 1 \mathrm{~min}$ ) involving anaerobic metabolism have been well investigated (Lericollais et al., 2009; Nicolas et al., 2005; Racinais et al., 2005a, 2005b; Souissi et al., 2004, 2007a, 2007b, 2008). During the Wingate test, peak power $\left(\mathrm{P}_{\text {peak }}\right)$ and mean power $\left(\mathrm{P}_{\text {mean }}\right)$ fluctuate with time-of-day, with the circadian acrophase (peak) at 17:24 $\pm 00: 36 \mathrm{~h}$ and 18:00 \pm 01:01 $\mathrm{h}$ (mean $\pm \mathrm{SD}$ ), respectively, and an amplitude of 7.6 \pm $0.8 \%$ and $11.3 \pm 1.1 \%$ (mean \pm SD), respectively (Souissi et al., 2004). Recently, we have shown a greater decrease in power during the Wingate test performed in the morning versus evening (Souissi et al., 2007a). Nonetheless, the underlying mechanisms of these time-of-day effects are still under discussion. Even if the circadian rhythm in core temperature is not necessarily the cause of the rhythm in muscle performance (Waterhouse et al., 2007), some studies have suggested that the simultaneous increases in central body temperature (assessed by oral or rectal measurements) and muscular power are causally related because the diurnal increase in central temperature may exert a beneficial passive warm-up effect (Bernard et al., 1998; Melhim, 1993; Racinais et al., $2005 \mathrm{a}, 2005 \mathrm{~b})$. Although the exact mechanisms to explain this relationship are not known, it has been suggested that higher body temperature may enhance metabolic reactions, increase the extensibility of connective tissue, reduce muscle viscosity, and increase conduction velocity of action potentials (Shephard, 1984). Indeed, Bergh and Ekblom (1979) demonstrated in warming and cooling experiments that maximal anaerobic power declines by $5 \%$ for every $1{ }^{\circ} \mathrm{C}$ decrease in muscle temperature.

According to Åstrand and Rodahl (1986), physical performance is optimal at a rectal temperature $\sim 38.5^{\circ} \mathrm{C}$, this being easier to reach in the late afternoon than morning. Thus, the optimal temperature level should be reached if the warm-up intensity and/or duration are increased in the morning. The idea that a substantial warm-up might negate any influence of diurnal variation is perhaps of greatest interest to applied sports scientists (Atkinson et al., 2005). While most studies have reported that an increase in muscle temperature following active warm-up has the potential to improve short-term performance (de Vries, 1959; Dolan et al., 1985; Thompson, 1958), a few studies have reported either no significant effect (Hawley et al., 1989; Pyke, 1968) or impaired performance (Margaria 
et al., 1971; Sargeant \& Dolan, 1987) following active warm-up. In the studies reporting a significant decrease in short-term performance following warm-up, it appears that the warm-up was either too intense and/or there was insufficient recovery between the active warm-up and subsequent task (Bishop, 2003).

Recently, Atkinson et al. (2005) and Racinais et al. (2005a) showed that the magnitude of the diurnal variation was larger than the magnitude of any warm-up effects on aerobic and anaerobic performances. In addition, Callard et al. (2000) observed that muscular force varied with time-of-day both at rest without prior exercise and during a continuous submaximal physical exercise of $24 \mathrm{~h}$. To our knowledge, the effects of active warm-up on diurnal fluctuations in anaerobic performance during efforts such as the $30 \mathrm{~s}$ Wingate have not been assessed. Therefore, the aim of the present study is to confirm that time of day has an effect on the results of the Wingate test and to examine if warm-up helps athletes to overcome the morning alteration in anaerobic scores. In practice, the Wingate test is never performed without any warming-up procedure. Therefore, we did not study the diurnal variation in the Wingate test without any warm-up. Either short sub-maximal sprints or cycling exercises with low resistance are usually performed $5 \mathrm{~min}$ before the Wingate test when it is the only test in a session. In many cases, the Wingate test is performed at the end of a testing session, after other protocols (e.g., aerobic tests, strength tests, vertical jump) that serve as preWingate warm-up procedures. Muscle temperature increases with the duration of warm-up exercise, but there is a ceiling of this effect beyond 15 min duration (Asmussen \& Böje, 1945). In a study by Högberg and Ljunggren (1947; cited by Åstrand \& Rodahl, 1986), running performances were better after a 15 min than after a 5 min warm-up, but there was no difference between the 15 and $30 \mathrm{~min}$ durations. Consequently, we tested the effects of a warm-up procedure (sub-maximal cycling at a power output around $50 \% \mathrm{VO}_{2} \max$ ) of short and long durations (5 versus $15 \mathrm{~min})$.

\section{METHODS}

\section{Subjects}

Twelve healthy male physical education students (23.5 \pm 3.1 yrs, $74.1 \pm 6.5 \mathrm{~kg}, 179.1 \pm 6.1 \mathrm{~cm}$; mean $\pm \mathrm{SD}$ ), not specially trained for either endurance or effort involved in sprint, volunteered to participate in the study. Subjects were fully informed of the protocol procedures and signed a consent form before participating. They were categorized either as "moderately morning type" $(n=4)$ or "neither type" ( $n=8)$ on the basis of their answers to the Horne and Östberg (1976) self-assessment 
questionnaire, which assesses morningness-eveningness. None of the study subjects was taking any vitamin or protein supplements. The present study complied with the ethical and procedural requirements of the journal for the conduct of human biological rhythm research (Portaluppi et al., 2008).

\section{Experimental Design}

Two weeks before the start of the experimental period, the subjects were familiarized with the cycle-ergometers and high-velocity cycling exercises to minimize learning effect during the course of the study. A few days after the familiarization sessions, subjects performed a medical stress test (ECG, heart rate, and arterial pressure monitoring during a progressive stress test beginning with $3 \mathrm{~min}$ at $50 \mathrm{~W}$ and $25 \mathrm{~W}$ increments every 2 min up to exhaustion) on a cycle-ergometer (LODE, Excalibur, Gröningen, The Netherlands) under the supervision of a physician at 14:00 h. The power corresponding to $50 \%$ of the power output in the last step of this aerobic stress test $\left(\mathrm{W}_{\text {aerobic }}\right)$ was chosen as the warm-up intensity. One week after this medical stress test, the subjects performed four test sessions in a randomized and balanced order over four days with only one test session a day, allowing a recovery period $\geq 36 \mathrm{~h}$. Two sessions were conducted in the morning (08:00 h) and two in the afternoon (18:00 h) with either a $5 \mathrm{~min}$ active warm-up (5-AWU) or a $15 \mathrm{~min}$ active warm-up (15-AWU).

Instructions about sleep and diet were given to the subjects before the experiment. Subjects were asked to keep their usual sleeping habits, with a minimum of $6 \mathrm{~h}$ sleep taken on the night preceding each test session. During the period of investigation, subjects were prohibited from consuming food, beverages, or any known stimuli (e.g., caffeine) or depressants (e.g., alcohol) that could possibly enhance or compromise alertness. Throughout the experimental period, participants were requested to maintain their habitual physical activity and to avoid strenuous activity during the $24 \mathrm{~h}$ before the test sessions. It was easy to control compliance with these instructions, because the participants were students who had exactly the same daily schedules in our institution. Subjects who were tested at 08:00 $\mathrm{h}$ were requested to report to the laboratory at 07:00 $\mathrm{h}$ in a fasting state. Only one glass $(15$ to $20 \mathrm{cl}$ ) of water was allowed, to avoid the effects of postprandial thermogenesis. After the morning session, unrestricted food intake was allowed. All subjects had the same standard isocaloric meal at 12:00 h, which concluded at least $5 \mathrm{~h}$ before the 18:00 h test. After that meal, only water was allowed ad libitum. Laboratory temperature was maintained at a constant of $20.4( \pm 1.1)^{\circ} \mathrm{C}$ during the entire experimental period in order to minimize potential effects of ambient temperature changes on the muscular system (Bergh \& Ekblom, 1979). 
The subjects' body temperature was continuously recorded throughout each test session using a rectal tube (Minimitter ${ }^{\circledR}$, Bend, Oregon, USA); values were collected at rest $\left(T_{\text {rest }}\right)$, at the end $\left(T_{\text {endWup }}\right)$ of the warm-up, and before $\left(\mathrm{T}_{\text {befWin }}\right)$ and at the end $\left(\mathrm{T}_{\text {endWin }}\right)$ of the Wingate test.

\section{Wingate Test}

The Wingate test was conducted on a friction-loaded cycle ergometer (Monark model 864 Crescent AB, Varberg, Sweden) interfaced with a microcomputer. The seat height and handlebars were adjusted appropriately for each subject. The Wingate test consisted of a $30 \mathrm{~s}$ maximal sprint against a constant resistance related to body mass $\left(0.087 \mathrm{~kg} \cdot \mathrm{kg}^{-1}\right.$ body mass) as proposed by Bar-Or (1987). The Wingate test began from a rolling start, at $60 \mathrm{rpm}$ against minimal resistance (weight basket supported). When a constant pedal rate of $60 \mathrm{rpm}$ was achieved, a countdown of "3-2-1-go!" was given, and the test resistance was applied. Subjects were verbally encouraged throughout the test to avoid pacing and to sustain a maximal effort throughout the test. Every second, power output was calculated by the computer and stored. The highest power output over $1 \mathrm{~s}\left(\mathrm{P}_{\text {peak }}\right)$ and the mean power $\left(\mathrm{P}_{\text {mean }}\right)$, corresponding to the ratio between total work done and time to do it (i.e., $30 \mathrm{~s}$ ), were recorded at the end of the test. The Fatigue Index (i.e., the percentage decrease in power output) was equal to the difference between the highest $\left(\mathrm{P}_{\text {peak }}\right)$ and lowest power $\left(\mathrm{P}_{\text {low }}\right)$ divided by the highest power:

$$
\text { Fatigue Index }=\left(\mathrm{P}_{\text {peak }}-\mathrm{P}_{\text {low }}\right) / \mathrm{P}_{\text {peak. }}
$$

\section{Statistical Analyses}

All statistical tests were processed using STATISTICA Software (StatSoft, France). The Shapiro-Wilk $W$-test of normality revealed the data were normally distributed. Temperature data were analyzed using a threefactor ANOVA (2 [warm-up duration] $\times 2$ [test time-of-day] $\times 4$ [measures] $)$ with repeated-measures on three factors. A two-way ANOVA [2 (Warm-up duration) $\times 2$ (Test time-of-day)] with repeated-measures on both factors was used to analyze the Wingate test performance data. When appropriate, significant differences between means were assessed using Tukey's post-hoc tests. We have used general multivariate analysis of variance using a general linear models (GLM) multivariate procedure to study the relationship between the time-of-day effect, the warm-up duration, and performance with temperature as a covariate. Statistical power was determined to be 0.80 for the sample size used at the $0.05 \alpha$ level. A probability level of 0.05 was selected as the criterion for statistical significance. 


\section{RESULTS}

\section{Temperature}

Rectal temperatures at rest, at the end of the warm-up, and before and at the end of the Wingate test in the two experimental conditions are shown in Figure 1. The effects of warm-up duration $\left(\mathrm{F}_{(1.7)}=14.5 ; p<\right.$ $0.01)$, time-of-day $\left(\mathrm{F}_{(1,7)}=37.6 ; p<0.001\right)$, and measure $\left(\mathrm{F}_{(3,21)}=146.6\right.$; $p<0.001)$ were significant with post-hoc tests showing that temperature improved significantly from morning to afternoon and from the baseline values after warm-up $(p<0.001)$. However, the interaction of warm-up $\times$ time-of-day $\times$ measure was not statistically significant $\left(\mathrm{F}_{(3.21)}=1.2 ; p>\right.$ 0.05), indicating that the time-of-day effects for all measurements did not depend on the warm-up durations. In fact, body temperature increased from the baseline values in the morning and afternoon $(p<0.001)$ after both warm-up durations. In addition, for both warm-up protocols, rectal temperature at all the measures increased from the morning to the afternoon $(p<0.001)$.

Changes from baseline of temperatures at the three times of measurements (i.e., end of warm-up and before and after the Wingate test) were greater with the 15-AWU than 5-AWU at both times-of-day ( $p<0.001$ ). These changes were the same at the three times of measurement with the 5-AWU and at the end of warm-up with the 15-AWU. However, with the 15-AWU, changes were significantly higher $(p<0.05)$ in the morning than afternoon, both before and after the Wingate test (see Table 1).

\section{Wingate Test}

Figures 2, 3, and 4 present the results of the Wingate test at 08:00 and 18:00 $\mathrm{h}$ for both warm-up conditions.

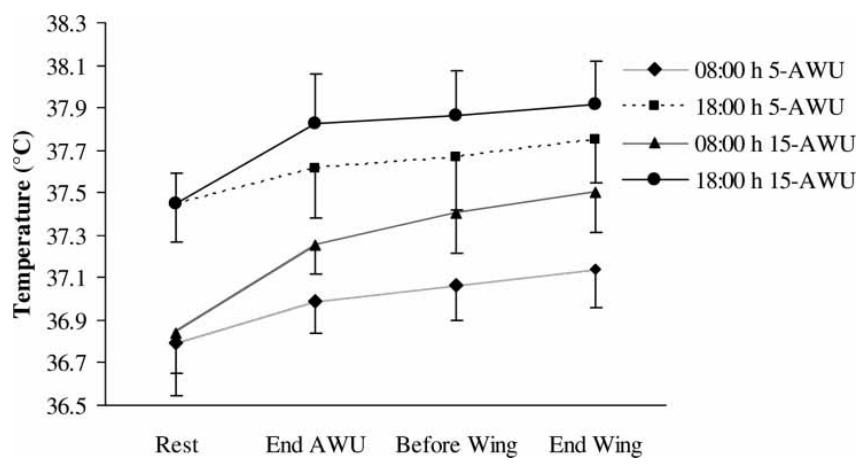

FIGURE 1 Mean and SD values for temperature $(\mathrm{n}=12)$ at 08:00 and 18:00 $\mathrm{h}$ after the $5 \mathrm{~min}$ active warm-up (5-AWU) and 15 min active warm-up (15-AWU) at rest, at the end of the warm-up (End AWU), and before (Before Wing) and at the end (End Wing) of the Wingate test. 
TABLE 1 Changes in temperature from baseline to end of warm-up (both 5 and 15 min) and before and after the Wingate test at 08:00 and 18:00 $\mathrm{h}$

\begin{tabular}{|c|c|c|c|c|}
\hline & \multicolumn{2}{|c|}{ Warm-up 5 min } & \multicolumn{2}{|c|}{ Warm-up 15 min } \\
\hline & $08: 00 \mathrm{~h}$ & 18:00 h & 08:00 h & $18: 00 \mathrm{~h}$ \\
\hline$\Delta \mathrm{T}_{\text {endWup }}\left({ }^{\circ} \mathrm{C}\right)$ & $0.19 \pm 0.1$ & $0.15 \pm 0.14$ & $0.41 \pm 0.15$ & $0.37 \pm 0.18$ \\
\hline$\Delta \mathrm{T}_{\text {befWin }}\left({ }^{\circ} \mathrm{C}\right)$ & $0.26 \pm 0.1$ & $0.21 \pm 0.15$ & $0.56 \pm 0.14$ & $0.41 \pm 0.17^{*}$ \\
\hline$\Delta \mathrm{T}_{\text {endWin }}\left({ }^{\circ} \mathrm{C}\right)$ & $0.34 \pm 0.1$ & $0.29 \pm 0.14$ & $0.66 \pm 0.16$ & $0.46 \pm 0.17^{*}$ \\
\hline
\end{tabular}

* Significant difference in comparison with 08:00 h after the same warm-up duration.

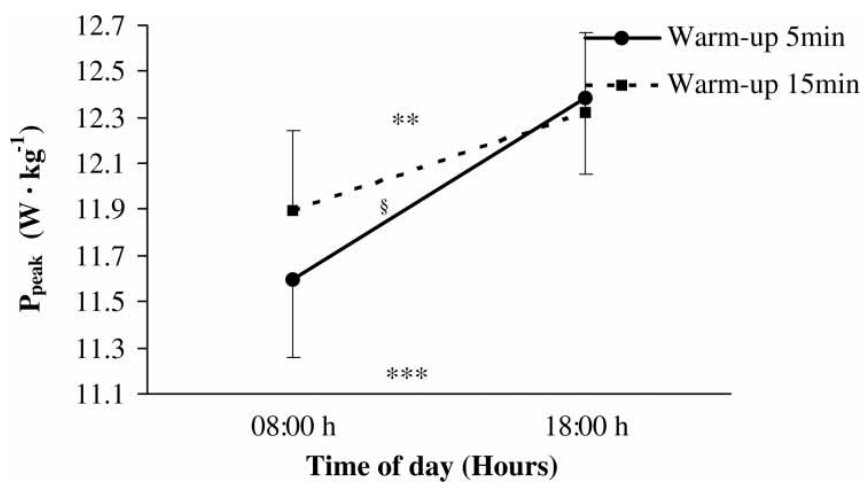

FIGURE 2 Mean and SE values for peak power $(\mathrm{n}=12)$ at 08:00 and 18:00 $\mathrm{h}$ after the 5 min active warm-up (5-AWU) and $15 \mathrm{~min}$ active warm-up (15-AWU). Significant differences in comparison with the afternoon $(* * p<0.01, * * * p<0.001)$. SSignificant difference in comparison with 5-AWU at the same time of day.

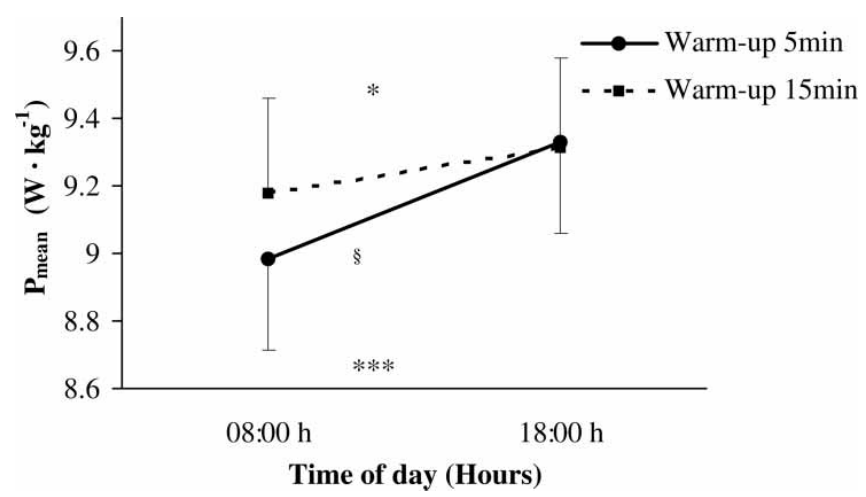

FIGURE 3 Mean and SE values for mean power $(\mathrm{n}=12)$ 08:00 and 18:00 $\mathrm{h}$ after the 5 min active warm-up (5-AWU) and $15 \mathrm{~min}$ active warm-up (15-AWU). Significant differences in comparison with the afternoon $\left(* * p<0.01,{ }^{* * *} p<0.001\right)$. SSignificant difference in comparison with 5-AWU at the same time of day.

\section{Peak Power}

The two-way ANOVA (warm-up $\times$ test time-of-day) for $\mathrm{P}_{\text {peak }}$ indicated that the time-of-day effect was significant $\left(\mathrm{F}_{(1.11)}=55.36, p<0.001\right)$ in 


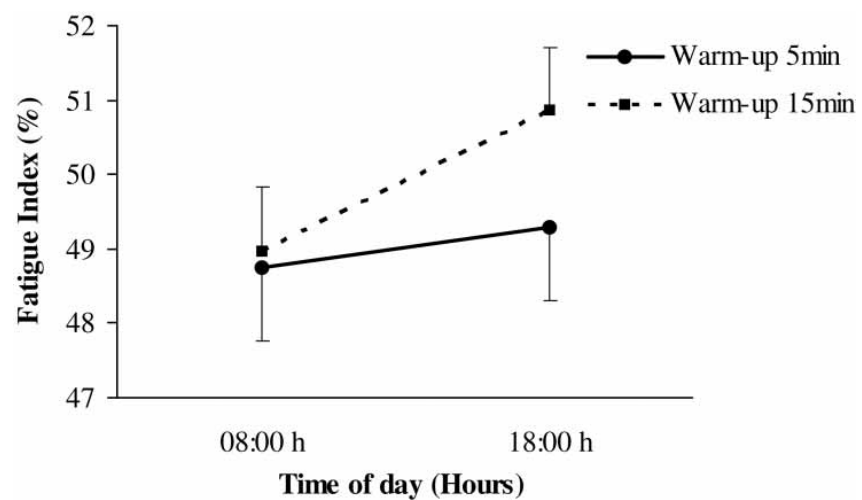

FIGURE 4 Mean and SE values for Fatigue Index $(\mathrm{n}=12)$ at 08:00 and 18:00 $\mathrm{h}$ after the $5 \mathrm{~min}$ active warm-up (5-AWU) and 15 min active warm-up (15-AWU).

contrast with the warm-up effect, which was not significant $(p>0.05)$. The warm-up $\times$ test time-of-day interaction was significant $\left(\mathrm{F}_{(1.11)}=6.48\right.$, $p<0.05)$. The post-hoc test revealed that $\mathrm{P}_{\text {peak }}$ was significantly higher in the morning with 15-AWU in comparison with 5-AWU $(p<0.05)$, but it was the same in the afternoon with both warm-up protocols. $\mathrm{P}_{\text {peak }}$ improved significantly from morning to evening $(p<0.001$ for 5-AWU and $p<0.01$ for 15-AWU). This improvement was greater $(p<0.05)$ after 5-AWU $(6.83[\mathrm{SD}=3.71] \%)$ than after 15-AWU (3.65 [SD = $2.58]^{\%}$ ). A GLM with temperatures ( $T_{\text {rest }}, T_{\text {endWup, }} T_{\text {befWin }}$, and $T_{\text {endWin }}$ ) as covariate showed that $\mathrm{P}_{\text {peak }}$ was independent of $\mathrm{T}_{\text {rest }}(\mathrm{F}=3.0, p>$ $\left.0.05, \eta^{2}=0.111\right), T_{\text {endWup }}\left(F=1.7, p>0.05, \eta^{2}=0.067\right)$, and $T_{\text {befWin }}$ $\left(\mathrm{F}=2.2, p>0.05, \eta^{2}=0.084\right)$. However, $\mathrm{T}_{\text {endWin }}$ influenced $\mathrm{P}_{\text {peak }}(\mathrm{F}=$ $\left.5.1, p<0.05, \eta^{2}=0.175\right)$.

\section{Mean Power}

The effect of warm-up protocol on $\mathrm{P}_{\text {mean }}$ was not significant $(p>$ 0.05) in contrast with a significant time-of-day effect $\left(\mathrm{F}_{(1.10)}=20.92, p<\right.$ $0.001)$. There was a significant interaction between warm-up and time-ofday $\left(\mathrm{F}_{(1.11)}=5.84, p<0.05\right)$. The post-hoc test revealed that $\mathrm{P}_{\text {mean }}$ improved significantly from morning to evening for 5-AWU $(p<0.001)$ and 15-AWU $(p<0.05)$. This improvement was greater $(p<0.05)$ for 5 AWU $\left(4.06[\mathrm{SD}=3.62]^{\%}\right)$ than 15-AWU $(1.59[\mathrm{SD}=1.84] \%) . \mathrm{P}_{\text {mean }}$ was significantly higher with 15 -AWU $(p<0.05)$ in the morning but was the same for both warm-up durations in the afternoon. A GLM with temperatures $\left(T_{\text {rest }}, T_{\text {endWup, }} T_{\text {befWin, and }} T_{\text {endWin }}\right)$ as covariate showed that $P_{\text {mean }}$ was independent of $\mathrm{T}_{\text {rest }}\left(\mathrm{F}=3.5, p>0.05, \mathrm{\eta}^{2}=0.128\right), \mathrm{T}_{\text {endWup }}(\mathrm{F}=$ 2.8, $\left.p>0.05, \eta^{2}=0.104\right)$, and $\mathrm{T}_{\text {befWin }}\left(\mathrm{F}=2.5, p>0.05, \eta^{2}=0.096\right)$. However, $\mathrm{T}_{\text {endWin }}$ influenced $\mathrm{P}_{\text {mean }}\left(\mathrm{F}=5.1, p<0.05, \eta^{2}=0.157\right)$. 


\section{Fatigue Index}

The Fatigue Index was independent of warm-up protocol and timeof-day, and there was no interaction between these factors $(p>0.05)$. A GLM with temperatures ( $T_{\text {rest }}, T_{\text {endWup, }} T_{\text {befWin, }}$ and $T_{\text {endWin }}$ ) as covariate showed the fatigue index was independent of $\mathrm{T}_{\text {rest }}(\mathrm{F}=0.02, p>$ $\left.0.05, \eta^{2}=0.001\right), T_{\text {endWup }}\left(F=0.03, p>0.05, \eta^{2}=0.002\right)$, and $T_{\text {befWin }}$ $\left(\mathrm{F}=2.1, \quad p>0.05, \quad \eta^{2}=0.08\right)$. However, $\mathrm{T}_{\text {endwin }}$ influenced fatigue index $\left(\mathrm{F}=7.0, p<0.05, \eta^{2}=0.226\right)$.

\section{DISCUSSION}

The main purpose of this study was to explore the effect of two durations of active warm-up on the diurnal fluctuations of muscle power. The results showed that the duration of the warm-up procedure (15 vs. 5 min) modified the magnitude of the diurnal fluctuation of anaerobic performances. Muscle power increased in the morning following the 15AWU in comparison with the 5-AWU. However, in the afternoon, anaerobic performances were the same after both AWU durations.

Concerning the technical procedure, cycle power measurement during the Wingate test has been shown to depend on the inertia of the flywheel (Lackomy, 1986). In the present study, as in our others (Souissi et al., 2004, 2007a, 2007b, 2008), this inertia was not taken into account for the power calculations. Nevertheless, as the present study aimed to compare the same test performed at different times, the possible error can be considered to be negligible in all cases.

In agreement with previous reports (Hill \& Smith, 1991; Melhim, 1993; Souissi et al., 2004, 2007a, 2007b, 2008), $P_{\text {peak }}$ and $P_{\text {mean }}$ were higher in the afternoon than morning. The diurnal variation in muscular power persisted after an AWU in accordance with the data of Racinais et al. (2005a), who observed that the diurnal variation in muscular power persisted after $12 \mathrm{~min} A W U$ at $50 \% \mathrm{VO}_{2} \max$ (i.e., the power corresponding to $\mathrm{VO}_{2}$ max). The effect of time of day upon $\mathrm{P}_{\max }$ was similar: $5.3 \%$ in the present study (average of 5 and 15-AWU data) versus $4.5 \%$ in Racinais et al. (2005). After 15 min warm-up, the significant effect ( $p<$ 0.01 ) of time-of-day was not negligible (4.47\%). The time-of-day effect was even larger $(6.8 \%)$ with the 5 -AWU protocol, but the difference was not significant. Consequently, time-of-day should be taken into account in the interpretation of the results of anaerobic tests in longitudinal (for example, before and after a training program) and transversal (e.g., comparison between athletes) studies, even when a warm-up procedure is included in the Wingate protocol.

The Fatigue Index is currently computed even if its physiological basis is questionable. The Fatigue Index was independent of time-of-day. 
Similarly, there was no effect of warm-up duration on this index. The reliability of the Fatigue Index is very low, as indicated by its test-retest coefficient of correlation in previous studies $(0.43<\mathrm{r}<0.74$; Bar-Or et al., 1987; Patton et al., 1985). Consequently, in the present study, it is likely that a diurnal variation due to an effect of warm-up duration on the Fatigue Index would have been masked by test-retest variations.

The results of the present study show that the duration of the warmup protocol modified the magnitude of the diurnal fluctuation of anaerobic performances. In the morning, $P_{\text {peak }}$ and $P_{\text {mean }}$ were significantly higher with the 15-AWU, whereas anaerobic performances were the same with the 5-AWU and 15-AWU in the afternoon. The longer the warm-up, the lower was the diurnal variation. These results are consistent in part with those of Arnett (2002), who increased swimmers' habitual warm-up in the morning and decreased their habitual warm-up in the afternoon. However, swimming performances were still superior in the afternoon. It should be noted that the increased warm-up in the morning stipulated by Arnett (2002) might have led to undue fatigue in the subsequent swimming time trial, as the effect of an increase in warm-up duration was not studied for the afternoon trials.

Warm-up intensity in the present study was $50 \% \mathrm{~W}_{\text {aerobic, }}$ because it has been shown that warm-up intensity $>60 \%$ of $\mathrm{W}_{\text {aerobic }}$ could alter performance during a subsequent cycling sprint (Sargeant \& Dolan, 1987). In the present study, warm-up procedures at $50 \% \mathrm{~W}_{\text {aerobic }}$ lasted 5 and $15 \mathrm{~min}$, as rectal temperature increases after $3-5 \mathrm{~min}$ of exercise and stabilizes after a minimum of $10 \mathrm{~min}$ (Saltin et al., 1968) at this exercise intensity. In practice, the Wingate test is never performed without any warm-up procedure. The results of the present study indicate that the usual warm-up procedures should be longer when the Wingate test is the only test in morning sessions.

Some authors (Bernard et al., 1998; Melhim, 1993) have hypothesized a causal link of the temporal fluctuation in core temperature among the factors frequently presented in the literature to explain the diurnal variation in muscular power. In agreement, our results showed a significant diurnal increase in rectal temperature at rest $\left(T_{\text {rest }}\right)$. As in the study by Racinais et al. (2005a), we observed a persistence of this diurnal variation after warm-up $\left(\mathrm{T}_{\text {endWup }}\right)$ and Wingate test $\left(\mathrm{T}_{\text {befWin }}\right.$ and $\left.\mathrm{T}_{\text {endWin }}\right)$ with both the 5-AWU and 15-AWU. In contrast with morning data, anaerobic performances $\left(\mathrm{P}_{\text {peak }}\right.$ and $\left.\mathrm{P}_{\text {mean }}\right)$ were independent of warm-up duration in the afternoon, in spite of the achievement of a higher rectal temperature with the 15-AWU. In addition, the GLM analysis showed that performances during the Wingate test depend only on $\mathrm{T}_{\text {endWin. These results }}$ suggest that the diurnal difference in core temperature was not the only explanation of the time-of-day effects on anaerobic performances. This observation is in accordance with the data of Martin et al. (1999), who 
observed the diurnal fluctuation in muscle strength persisted despite an artificial heating of the adductor pollicis muscle by $5^{\circ} \mathrm{C}$ at $07: 00 \mathrm{~h}$. However, a ceiling of the temperature effect upon maximal power is another explanation. For example, the optimal rectal and muscle temperatures should be, respectively, $\sim 38.5^{\circ} \mathrm{C}$ and $39^{\circ} \mathrm{C}$ according to Åstrand and Rodahl (1986). Bergh and Ekblom (1979) demonstrated that power output, jumping, and sprinting performances decrease by $5 \%$ for every $1^{\circ} \mathrm{C}$ decline in muscle temperature in warming and cooling experiments for muscle temperatures between 30 and $39^{\circ} \mathrm{C}$. More recently, Sargeant and Dolan (1987) observed that maximal power output at high pedaling rate increases by approximately $10 \%$ per $1{ }^{\circ} \mathrm{C}$ (muscle temperature) after 45 min of leg immersion in water baths at 44,18 , and $12^{\circ} \mathrm{C}$, with no indication of a ceiling effect. However, muscle temperature after immersion depends on muscle depth, and it is difficult to estimate the optimal muscle temperature for high velocity cycling exercises and its corresponding rectal temperature level from the Sargeant and Dolan study.

In conclusion, the present study confirms that time-of-day and warmup procedures have significant effects on anaerobic performances. The effect on warm-up duration on rectal temperature after warm-up and the Wingate test was independent of time-of-day, whereas its effect on anaerobic performance was observed in the morning sessions, only. Either the observed variation in core temperature was not the only explanation of these results, or there was a ceiling of the effect of temperature on muscle performance. Athletes should increase the AWU duration in the morning in order to improve their anaerobic performances. On the other hand, the results of the present study suggest that short warm-up procedures are sufficient to alter Wingate test performance in the afternoon.

\section{ACKNOWLEDGMENTS}

The authors wish to express their sincere gratitude to all the participants for their maximal effort and cooperation.

\section{DECLARATION OF INTEREST}

The authors report no conflict of interest. The authors alone are responsible for the content and writing of the article.

\section{REFERENCES}

Arnett MG. (2002). Effects of prolonged and reduced warm-ups on diurnal variation in body temperature and swim performance. J. Strength Cond. Res. 16:256-261.

Asmussen E, Böje O. (1945). Body temperature and capacity for work. Acta Physiol. Scand. 10:1-22. Åstrand PO, Rodahl K. (1986). Textbook of work physiology. Physiological bases of exercise. 3rd ed.

New York: McGraw-Hill Book Co., pp. 596-614. 
Atkinson G, Todd C, Reilly T, Waterhouse J. (2005). Diurnal variation in cycling performance: Influence of warm-up. J. Sports Sci.23:321-329.

Bar-Or O. (1987). The Wingate anaerobic test: An update on methodology, reliability and validity. Sports Med. 4:381-394.

Bergh U, Ekblom B. (1979). Influence of muscle temperature on maximal muscle strength and power output in human skeletal muscles. Acta Physiol. Scand.107:33-37.

Bernard T, Giacomoni M, Gavarry O, Seymat M, Falgairette G. (1998). Time-of-day effects in maximal anaerobic leg exercise. Eur. J. Appl. Physiol. 77:133-138.

Bishop D. (2003). Performance changes following active warm-up and how to structure the warm-up. Sports Med. 33:484-498.

Callard D, Davenne D, Gauthier A, Lagarde D, Van Hoecke J. (2000). Circadian rhythms in human muscular efficiency: Continuous physical exercise versus continuous rest. A crossover study. Chronobiol. Int. 17:693-704.

de Vries HA. (1959). Effects of various warm-up procedures on 100-yard times of competitive swimmers. Res. Q. Exerc. Sport 30:11-22.

Dolan P, Greig C, Sargeant AJ. (1985). Effect of active and passive warm-up on maximal short-term power output of human muscle [abstract]. J. Physiol. 365:P74.

Hawley JA, Williams MM, Hamling GC. (1989). Effects of a task specific warm-up on anaerobic power. Br. J. Sports Med. 23:233-236.

Hill DW, Smith JC. (1991). Circadian rhythm in anaerobic power and capacity. Can. J. Sport Sci. $16: 30-32$.

Horne JA, Östberg O. (1976). A self-assessment questionnaire to determine morningness-eveningness in human circadian rhythms. Int. J. Chronobiol. 4:97-110.

Lakomy HK. (1986). Measurement of work and power output using friction-loaded cycle ergometers. Ergonomics. 29: 509-517.

Lericollais R, Gauthier A, Bessot N, Sesboüé B, Davenne D. (2009). Time-of-day effects on fatigue during a standard anaerobic test in well-trained cyclists. Chronobiol. Int. 26:1622-1635.

Margaria R, di Prampero PE, Aghemo P. (1971). Effect of a steady-state exercise on maximal anaerobic power in man. J. Appl. Physiol. 30:885-889.

Martin A, Carpentier A, Guissard N, Van Hoecke J, Duchateau J. (1999). Effect of time of day on force variation in a muscle. Muscle Nerve 22:1380-1387.

Melhim AF. (1993). Investigation of circadian rhythms in peak power and mean power of female physical education students. Int. J. Sports Med. 14:303-306.

Nicolas A, Gauthier A, Bessot N, Moussay S. Davenne D. (2005). Time-of-day effects on myoelectric and mechanical properties of muscle during maximal and prolonged isokinetic exercise. Chronobiol. Int. 22:997-1011.

Patton JF, Murphy MM, Frederick FA. (1985). Maximal power outputs during the Wingate Anaerobic Test. Int. J. Sports Med. 6:82-85.

Portaluppi F, Touitou Y, Smolensky MH. (2008). Ethical and methodological standards for laboratory and medical biological rhythm research. Chronobiol. Int. 25:999-1016.

Pyke FS. (1968). The effect of preliminary activity on maximal motor performance. Res. Q. Exerc. Sport. 39:1069-1076.

Racinais S, Blonc S, Hue O. (2005a). Effects of active warm-up and diurnal increase in temperature on muscular power. Med. Sci. Sports Exerc. 37:2134-2139.

Racinais S, Blonc S, Jonville S, Hue O. (2005b). Time of day influences the environmental effects on muscle force and contractility. Med. Sci. Sports Exerc. 37:256-261.

Saltin B, Gagge AP, Stolwijk JA. (1968). Muscle temperature during submaximal exercise in man. J. Appl. Physiol. 25: 679-688.

Sargeant AJ, Dolan P. (1987). Effect of prior exercise on maximal short term power output in humans. J. Appl. Physiol. 63:1475-1480.

Shephard RJ. (1984). Sleep, biorhythms and human performance. Sports Med. 1:11-37.

Souissi N, Gauthier A, Sesboüé B, Larue J, Davenne D. (2004). Circadian rhythms in two types of anaerobic cycle leg exercise: Force-velocity and 30-s Wingate tests. Int. J. Sports Med. 25:14-19.

Souissi N, Bessot N, Chamari K, Gauthier A, Sesboüé B, Davenne D. (2007a). Effect of time of day on aerobic contribution to the 30-s Wingate test performance. Chronobiol. Int. 24:739-748.

Souissi N, Souissi H, Sahli S, Tabka T, Dogui M, Ati J, Davenne D. (2007b). Effect of Ramadan on the diurnal variation in short-term high power output. Chronobiol. Int. 24:991-1005. 
Souissi N, Souissi M, Souissi H, Chamari K, Tabka Z, Dogui M, Davenne D. (2008). Effect of time of day and partial sleep-deprivation on short-term, high power output. Chronobiol. Int. 25:1062-1076.

Thompson H. (1958). Effect of warm-up upon physical performance in selected activities. Res. Q. Exerc. Sport 29:231-246.

Waterhouse J, Aizawa S, Nevill A, Edwards B, Weinert D, Atkinson G, Reilly T. (2007). Rectal temperature, distal sweat rate, and forearm blood flow following mild exercise at two phases of the circadian cycle. Chronobiol. Int. 24:63-85. 